\title{
Indicadores de saúde em escolares com e sem Transtorno do Desenvolvimento da Coordenação - TDC
}

\author{
Renata Capistrano, Juliano Maestri Alexandre, Thais Silva Beltrame \\ Universidade do Estado de Santa Catarina - UDESC, Florianópolis, SC, Brasil.
}

\begin{abstract}
Resumo: Objetivo: Este estudo objetivou investigar os indicadores de saúde de crianças com e sem Transtorno do Desenvolvimento da Coordenação - TDC. Método: Participaram do estudo 83 crianças, com idade de 8 a 10 anos, de escolas particulares de São José-SC. Para avaliar o desempenho motor, foi utilizada a Movement Assessment Battery for Children Second Edition - MABC-2; os dados das variáveis referentes ao status do peso corporal foram obtidos por meio de um estadiômetro Sanny, balança Tânita, plicômetro e trena antropométrica Cescorf. O nível de atividade física foi mensurado com o questionário Dia Típico de Atividades Físicas e Alimentação - DAFA. Para a resistência cardiorrespiratória, foi realizado o Teste de 6 minutos. Os dados coletados para a realização das análises estatísticas descritivas (médias, mínimos, máximos, desvio padrão, frequências) e inferenciais (Teste Qui-quadrado, Exato de Fisher, Teste T independente, Curva ROC - Receiver Operating Characteristic) foram tabulados no SPSS. A significância estatística adotada foi de $\mathrm{p} \leq 0,05$. Resultados: Observou-se que os meninos apresentaram prevalência superior para o risco/dificuldade motora (41,5\%) em relação às meninas (19,5\%). As crianças com TDC apresentaram IMC superior $\left(20,2 \mathrm{~kg} / \mathrm{m}^{2}\right)$ e resistência cardiorrespiratória inferior $(673,2 \mathrm{~m})$, em relação às crianças sem o transtorno $\left(18,5 \mathrm{~kg} / \mathrm{m}^{2}\right.$ e $\left.719,1 \mathrm{~m}\right)$. Na relação entre a resistência cardiorrespiratória e a presença do TDC, a área sob a curva ROC foi de 0,327 e o ponto de corte de 654,5 m. Conclusão: Os resultados reportam a necessidade de intervenções voltadas à adoção de um estilo de vida ativo, a fim de minimizar ou reverter o desfecho encontrado.
\end{abstract}

Palavras-chave: Transtorno do Desenvolvimento da Coordenação, Crianças, Saúde.

\section{Health status indicators in schoolchildren with and without Developmental Coordination Disorder - DCD}

\begin{abstract}
Objective: This study aimed to investigate the health status indicators in children with and without Developmental Coordination Disorder - DCD. Method: The study included 83 children aged 8-10 years of private schools at São José-SC. To evaluate the motor performance, Movement Assessment Battery for Children Second Edition - MABC-2 was used; the variables related to the body weight status were assessed by a Sanny stadiometer, Tanita scale, Cescorf caliper and anthropometric tape. The level of physical activity was measured with the Dia Típico de Atividades Físicas e Alimentação - DAFA questionnaire. The 6 minutes test was performed for cardiorespiratory endurance. The collected data to perform the descriptive (average, minimum, maximum, standard deviation, frequency) and inferential (Chi-square test, Fisher exact test, test independent T, ROC - Receiver Operating Characteristic) statistical analyzes were tabulated using SPSS. The assumed statistical significance was $p \leq 0.05$. Results: Boys had higher prevalence of motor risk/difficulty (41.5\%) compared to girls (19.5\%). The children with DCD presented higher BMI $(20.2 \mathrm{~kg} / \mathrm{m} 2)$ and lower cardiorespiratory endurance $(673.2 \mathrm{~m})$ when compared to children without the disorder $(18.5 \mathrm{~kg} / \mathrm{m} 2$ e $719.1 \mathrm{~m})$. Regarding the presence of DCD, the area under the ROC curve was 0.327 and the cutoff was $654.5 \mathrm{~m}$. Conclusion: The results indicate the need for interventions aiming the adoption of an active lifestyle to minimize or reverse the outcome found.
\end{abstract}

Keywords: Developmental Coordination Disorder, Child, Health.

Autor para correspondência: Renata Capistrano, Centro de Ciências da Saúde e do Esporte, Rua Pascoal Simone, 358, Coqueiros, CEP 88080-350, Florianópolis, SC, Brasil, e-mail: recapis@gmail.com

Recebido em Jan. 29, 2015; 1ª Revisão em Abr. 11, 2015; 2ª Revisão em Jun. 8, 2015; Aceito em Jul. $29,2015$. 


\section{Introdução}

As investigações em desenvolvimento motor têm se tornando cada vez mais frequentes e se legitimam, uma vez que possibilitam identificar como a maioria das pessoas se desenvolve em relação ao seu movimento corporal, permitindo a realização de diagnósticos e intervençōes (PAYNE; ISAACS, 2007). De acordo com teorias desenvolvimentistas, espera-se que crianças em condiçôes adequadas de desenvolvimento apresentem um crescente domínio do aparato motor ao longo dos anos. No entanto, em alguns casos, verifica-se a presença de dificuldades motoras que inibem ou impedem a execução de funções da vida diária ou conquistas acadêmicas, sendo denominadas tais dificuldades de Transtorno do Desenvolvimento da Coordenação (TDC).

O TDC é caracterizado por um comprometimento do desempenho de atividades diárias e acadêmicas, sem a presença de condiçóes clínicas conhecidas, como a paralisia cerebral ou a distrofia muscular, tendo por base a idade cronológica e a inteligência (AMERICAN..., 2014). Crianças com dificuldade motora apresentam prejuízos no desempenho funcional por não serem proficientes na realização de tarefas cotidianas, como atividades de cuidado pessoal e escolares, nas quais demonstram resultados abaixo $\mathrm{da}$ média para a sua faixa etária (WANG; MONTEIRO; POPKIN, 2002). São também caracterizadas como "desajeitadas", por apresentarem coordenação motora pobre, problemas de ritmo, dificuldades em tarefas de esporte, lazer e interação social (GIBBS; APPLETON; APPLETON, 2007). Diante disso, pesquisas internacionais apontam que crianças com dificuldades de movimento não se engajam em situaçôes que demandam exigências físicas e se afastam gradativamente das mesmas (HAGA, 2008; WU et al., 2010; GREEN et al., 2011), apresentando uma menor participação em atividades organizadas, como jogos com regras, o que contribui para uma baixa aptidão física, resultando em maiores riscos para o desenvolvimento de sobrepeso e obesidade (TSIOTRA et al., 2006; HANDS; LARKIN, 2006; HAGA, 2008; CAIRNEY et al., 2010; WU et al., 2010; ZHU; WU; CAIRNEY, 2011; ZHU et al., 2014; HENDRIX; PRINS; DEKKERS, 2014).

No Brasil, poucos estudos foram encontrados fazendo essas relaçôes, (MIRANDA; BELTRAME; CARDOSO, 2011; MARRAMARCO et al., 2012), fato justificado pela baixa disseminação do termo no país e pela inexistência de instrumentos validados para a população brasileira, para a identificação do TDC. Além disso, as poucas pesquisas não permitem inferir resultados conclusivos acerca da direção das associações. Miranda, Beltrame e Cardoso (2011), ao analisarem escolares de 7 a 10 anos, com e sem TDC, não encontraram associação entre o estado nutricional e o desempenho motor. Marramarco et al. (2012), por sua vez, verificaram associação entre o estado nutricional e o desempenho motor de escolares de 5 a 10 anos. Na relação entre a presença de TDC e o nível de atividade física e aptidão física, não foi evidenciada a presença de estudos nacionais acerca desta temática. Diante disso, no intuito de fornecer subsídios para futuras pesquisas sobre o tema, bem como ampliar os dados sobre este transtorno entre crianças brasileiras, a fim de predizer as condiçôes de saúde dos escolares, este estudo objetivou investigar os indicadores de saúde em escolares com e sem Transtorno do Desenvolvimento da Coordenação - TDC.

\section{Método}

\subsection{Participantes do estudo}

Participaram do estudo, 83 escolares de ambos os sexos, com idade de 8 a 10 anos, de duas escolas particulares localizadas no município de São José-SC. Os escolares nessa faixa etária contemplavam as séries do $3 .^{\circ}$ ao $5 .^{\circ}$ ano do Ensino Fundamental, nos períodos, matutino e vespertino.

A escolha das instituiçóes foi feita de forma intencional devido à facilidade de acesso dos pesquisadores. A seleçáo dos participantes foi não probabilística, conforme a disponibilidade dos alunos para participação na pesquisa. Os critérios de inclusáo considerados foram: intenção dos escolares para participação voluntária no estudo; faixa etária estabelecida (8 a 10 anos); apresentação da autorização dos pais ou responsáveis por meio da assinatura do Termo de Consentimento Livre e Esclarecido (TCLE); ausência de deficiências físicas nos membros inferiores ou superiores e/ou deficiências mentais, indicadas pelos professores regentes e professores de Educação Física.

\subsection{Instrumentos}

Para avaliação motora, utilizou-se a Movement Assessment Battery for Children - MABC-2. Trata-se de um teste motor com três conjuntos de tarefas (Destreza Manual, Lançar e Receber, e Equilíbrio), apropriadas para as faixas etárias específicas: Faixa um (3 a 6 anos); Faixa dois (7 a 10 anos) e Faixa três (11 a 16 anos). Para cada faixa etária, são estabelecidas tarefas diferenciadas, de acordo com os níveis diferentes de complexidade. Neste estudo, 
especificamente, foram utilizados os testes da faixa etária 2 (7 a 10 anos). Os resultados da avaliação são calculados pelos valores do MABC-2 Total, comparados na tabela de percentis, presentes no protocolo do teste. As crianças cuja pontuação total foi abaixo do $5 .^{\circ}$ percentil, devem ser consideradas com um indicativo de dificuldade motora; valores entre o $5 .^{\circ}$ e o $15 .^{\circ}$ percentil indicam que a criança tem um grau de dificuldade motora (risco de dificuldade motora), e valores acima do $15 .^{\circ}$ percentil indicam um desenvolvimento motor normal.

As medidas de massa corporal e estatura, utilizadas para o cálculo do Índice de Massa Corporal $\left(\mathrm{IMC}=\mathrm{kg} / \mathrm{m}^{2}\right)$ foram padronizadas de acordo com Alvarez e Pavan (1999). As medidas de massa corporal foram realizadas por meio de uma balança marca Tânita e a estatura corporal foi medida por meio de um estadiômetro marca Sanny.

A circunferência da cintura, bem como a abdominal, foram aferidas seguindo a padronização de Martins e Lopes (2003). As dobras cutâneas tricipital e subescapular foram avaliadas por um compasso Cescorf seguindo a padronização de Benedetti, Pinho e Ramos (1999). Para o cálculo do Percentual de Gordura Corporal, foram utilizadas as equaçóes de Slaughter et al. (1988), apropriadas para crianças e adolescentes.

O teste de resistência cardiorrespiratória faz parte do Manual de Aplicação de Medidas, Testes, Normas e Critérios de Avaliação do Projeto Esporte Brasil - PROESP-BR (GAYA, 2009).

O nível de atividade física foi mensurado por meio do questionário Dia Típico de Atividades Físicas e Alimentação - DAFA (BARROS et al., 2007).

Para avaliar o nível socioeconômico dos participantes do estudo, foi utilizado o questionário socioeconômico da Associação Brasileira de Empresas de Pesquisa (ASSOCIAÇÃO..., 2012), sendo dividido em três classes: "Alta" ("A" + "B"); "Média" ("C"); "Baixa" (“D” + "E”).

\subsection{Procedimento de coleta de dados}

Esta pesquisa foi realizada de acordo com a resoluçấo n. ${ }^{\circ}$ 466/2012 do Conselho Nacional de Saúde (CNS), aprovada em seus aspectos éticos e metodológicos pelo Comitê de Ética e Pesquisa em Seres Humanos da Universidade do Estado de Santa Catarina (CEPSH/UDESC), sob o número de protocolo 16449913.8.0000.0118. O foco do estudo foi a avaliação de escolares com idade entre 8 e 10 anos, e, conforme as informaçóes da Direção da Escola, essa faixa etária contempla as séries do 3. ${ }^{\circ}$ ao $5 .^{\circ}$ ano do Ensino Fundamental.

Durante as visitas às turmas, foi feito o convite aos escolares da faixa etária pretendida para participação no estudo, sendo entregue aos mesmos o TCLE, para que fosse assinado pelos pais ou responsáveis, para que autorizassem a participação das crianças. Após os recebimentos dos termos de consentimento assinados, as coletas de dados se iniciaram.

Todas as avaliaçóes foram realizadas na escola, em ambientes iluminados e longe de interferências (sala de aula ampla e pátio da escola), com turno e horários combinados previamente com a Direção Pedagógica. Todos os pesquisadores receberam capacitaçóes anteriormente às coletas, a fim de padronizar os procedimentos das avaliaçôes.

A duração total de aplicação das avaliaçóes durou cerca de uma hora e meia por aluno, variando conforme a habilidade dos escolares no desempenho das tarefas. A identificação dos participantes foi registrada por números, de modo a preservar os escolares e atender aos princípios éticos.

O retorno das informaçóes à escola e aos alunos (e seus responsáveis) aconteceu por meio de relatórios individuais e gerais, nos quais foram descritos os objetivos do estudo, os resultados das avaliaçóes e as sugestôes.

\subsection{Análise estatística}

Após a coleta de dados, os mesmos foram tabulados no programa Statistical Package for Social Sciences - SPSS, versão $20.0^{\circ}$. A análise descritiva foi realizada por meio de médias, valores mínimos e máximos, frequências e desvios padrão. Para verificação da normalidade dos dados, foi utilizado o Teste de Kolmogorov-Smirnov.

Quanto à estatística inferencial, para verificação das associaçôes entre as variáveis categóricas (sexo/classificação motora; nível socioeconômico/classificação motora), utilizou-se o Teste Qui-quadrado ou Exato de Fisher, quando necessário, com cálculo do ajuste residual $\geq 2$. Para a análise dos dados, as crianças com risco e dificuldade motora foram alocadas no mesmo grupo, e o segundo grupo foi formado pelas crianças sem dificuldade motora, sendo nomeados como: "Risco/Dificuldade motora", "Sem dificuldade motora". Para a comparaçáo entre os grupos em relação às variáveis numéricas, foi utilizado o Teste T de Student. Além disso, foi calculado o Efeito $d$ de Cohen. A significância estatística adotada foi de $\mathrm{p} \leq 0,05$. 
Os pontos de corte para a Resistência Cardiorrespiratória foram determinados por meio da curva ROC (Receiver Operating Characteristic), identificando a área total sob a curva ROC e o ponto de coorte com melhor sensibilidade e especificidade.

\section{Resultados}

Participaram do estudo, 83 crianças com média de idade de $8,9( \pm 0,74)$ anos, sendo $49,4 \%(\mathrm{n}=41)$ do sexo masculino e $50,6 \%(\mathrm{n}=42)$ do feminino. Em relação ao nível socioeconômico, 81,9\% dos participantes pertencem à classe média e $18,1 \%$ à classe alta.

A prevalência de dificuldade motora e risco para dificuldade motora foi de $9,8 \%$ e $20,7 \%$, respectivamente, e sem dificuldade de movimento foi de $69,5 \%$. Ao se verificarem as diferenças entre meninos e meninas com relação aos domínios da classificação motora, as meninas apresentaram escores padrão superiores para a destreza manual $(20,93)$ e equilíbrio $(47,09)$, em comparação aos meninos, com $(16,43)$ e $(43,51)$, respectivamente; porém, estas diferenças não foram significantes ( $\mathrm{p}$-valor $=0,13$; $0,09)$. Para o domínio 'lançar e receber', foram evidenciadas diferenças significantes ( $\mathrm{p}$-valor $=0,04$ ), sendo que os meninos apresentaram maiores médias nas habilidades $(56,65)$, quando comparados às meninas $(45,02)$.

A associação entre o sexo e a classificação motora demonstrou que os escolares do sexo masculino apresentaram maior prevalência de risco/dificuldade motora $(41,5 \%)$ em relação às meninas $(19,5 \%)$ $\left(\mathrm{X}^{2}=4,66 ; \mathrm{p}=0,03\right)$. Entre o nível socioeconômico e a classificação motora, náo foi encontrada associaçáo significante $\left(X^{2}=5,02 ; p=0,08\right)$.
$\mathrm{Na}$ Tabela 1, são apresentados os valores referentes à comparação entre os escolares com risco/dificuldade motora e sem dificuldade motora, quanto aos indicadores de saúde. Os resultados mostram que houve diferença significante para o IMC $\left[\mathrm{t}_{(78)}=2,00\right.$; $\mathrm{p}=0,04]$ e para a resistência cardiorrespiratória $\left[\mathrm{t}\left({ }_{77}\right)=2,22 ; \mathrm{p}=0,02\right]$, sendo que as crianças sem dificuldade motora apresentaram valor inferior para o IMC $\left(18,5 \mathrm{~kg} / \mathrm{m}^{2}\right)$ em relação àquelas com risco/dificuldade motora $\left(20,2 \mathrm{~kg} / \mathrm{m}^{2}\right)$, e valores superiores para a resistência cardiorrespiratória $(719,1 \mathrm{~m})$ em relação aos participantes com risco/dificuldade motora $(673,2 \mathrm{~m})$. Ressalta-se que, conforme o Coeficiente $d$ de Cohen $(0,44)$, existe um médio efeito do Risco/dificuldade motora sobre o IMC.

Quanto à relação entre o IMC e a presença da dificuldade motora (Tabela 2), a área sob a curva ROC foi de 0,620 , sendo que esse resultado náo foi significativo $(\mathrm{p}=0,08)$. Na relação entre a resistência cardiorrespiratória e a presença de dificuldade motora (Tabela 2), nota-se que a área sob a curva ROC foi de 0,327 , sendo que o ponto de corte de $654,5 \mathrm{~m}$ ou menos apresenta o equilíbrio mais adequado entre a sensibilidade e a especificidade. Neste, o poder da resistência cardiorrespiratória para classificar corretamente crianças com dificuldade motora é de $47,8 \%$ (sensibilidade), enquanto que o poder desse mesmo componente da aptidão física para classificar corretamente os escolares sem dificuldade motora é de $82,1 \%$ (especificidade) (Figura 1).

\section{Discussão}

Embora, haja limitaçóes neste estudo, como o número de indivíduos, a presente investigaçáo contribui para o corpo de conhecimento na área do

Tabela 1. Comparação das médias nas avaliações de saúde conforme a classificação motora de escolares $(n=83)$.

\begin{tabular}{|c|c|c|c|c|c|}
\hline Indicadores de Saúde & $\begin{array}{c}\text { Sem Dificuldade } \\
\text { Motora }\end{array}$ & $\begin{array}{c}\text { Com Risco/ } \\
\text { Dificuldade Motora }\end{array}$ & $\mathbf{T}$ & $\mathbf{P}$ & $\begin{array}{c}\text { Coeficiente } d \\
\text { de Cohen }\end{array}$ \\
\hline & Média(dp) & Média(dp) & & & \\
\hline $\begin{array}{l}\text { Índice de Massa Corporal - } \\
\mathrm{IMC}_{(\mathrm{kg} / \mathrm{m})}^{2}\end{array}$ & $18,51(2,84)$ & $20,21(4,62)$ & 2,01 & $0,04 *$ & 0,44 \\
\hline $\begin{array}{l}\text { Somatório das Dobras } \\
(\mathrm{SE}+\mathrm{TR})\end{array}$ & $25,66(10,57)$ & $27,63(10,23)$ & 0,75 & 0,45 & \\
\hline Circunferência da Cintura $_{(\mathrm{cm})}$ & $62,16(7,10)$ & $64,00(7,91)$ & 1,00 & 0,31 & \\
\hline Circunferência Abdominal $_{(\mathrm{cm})}$ & $67,41(8,70)$ & $69,95(9,96)$ & 1,12 & 0,26 & \\
\hline $\begin{array}{l}\text { Resistência } \\
\text { Cardiorrespiratória }_{(\mathrm{m})}\end{array}$ & $719,1(86,2)$ & $673,2(71,9)$ & 2,22 & $0,02 *$ & 0,57 \\
\hline Nível de Atividade Física & $28,90(13,0)$ & $28,40(10,4)$ & 0,16 & 0,87 & \\
\hline
\end{tabular}


Tabela 2. Área sobre a curva ROC, nível de significância ( $(p)$, pontos de corte, sensibilidade e especificidade do IMC e resistência cardiorrespiratória associada à presença da dificuldade motora em escolares $(\mathrm{n}=83)$.

\begin{tabular}{|c|c|c|c|c|c|c|}
\hline Variáveis & $\begin{array}{l}\text { Área } \\
\text { curva } \\
\text { ROC }\end{array}$ & $\begin{array}{c}\text { IC } 95 \% \\
\text { Área curva } \\
\text { ROC }\end{array}$ & $P$ & $\begin{array}{l}\text { Ponto de } \\
\text { Corte }\end{array}$ & Sensibilidade & Especificidade \\
\hline $\begin{array}{l}\text { Risco/Dificuldade } \\
\text { Motora } \\
\text { IMC } \mathrm{kg} / \mathrm{m}^{2}\end{array}$ & 0,620 & $0,477-0,763$ & 0,08 & & & \\
\hline $\begin{array}{l}\text { Risco/Dificuldade } \\
\text { Motora }\end{array}$ & 0,327 & $0,197-0,457$ & $0,01 *$ & $<=654,5$ & 47,8 & 82,1 \\
\hline $\begin{array}{l}\text { Resistência } \\
\text { Cardiorrespiratória } m\end{array}$ & & & & & & \\
\hline
\end{tabular}

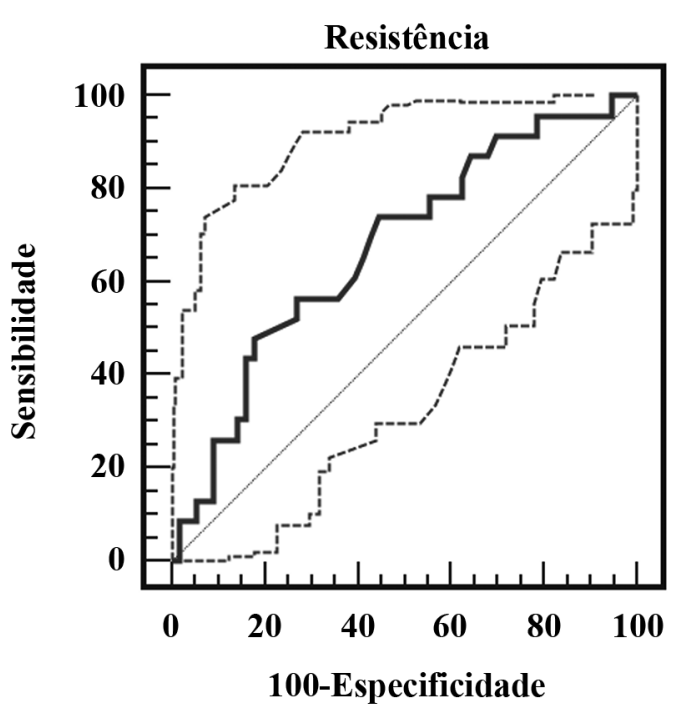

Figura 1. Curva ROC da resistência cardiorrespiratória para discriminar a ocorrência da dificuldade motora em escolares. Florianópolis.

desempenho motor, demonstrando que os meninos apresentaram maior prevalência de risco/dificuldade motora em relação às meninas e que os escolares com TDC demonstraram IMC superior e resistência cardiorrespiratória inferior, quando comparados aos seus pares sem o transtorno.

Ao avaliar o desempenho motor dos escolares analisados, verificou-se que 9,8\% deles apresentaram dificuldade motora, valor semelhante aos percentuais encontrados no Brasil e elevado quando comparado aos divulgados em pesquisas internacionais. Na Inglaterra, foram avaliadas 4.331 crianças, sendo que $4,4 \%$ apresentaram o transtorno (GREEN et al., 2011). No Canadá, 590 crianças foram avaliadas, sendo que 7,8\% apresentaram o TDC (CAIRNEY et al., 2012). No Brasil, Silva e Beltrame (2013), França (2009) encontraram $11,1 \%$ e $10,8 \%$ de crianças com TDC, respectivamente, na cidade de Florianópolis-SC. Salientamos a necessidade de atenção ao grupo de alunos classificados com risco ou dificuldade motora, orientando os professores de Educação Física sobre a importância da elaboração de estratégias nas aulas que potencializem e estimulem as capacidades motoras destas crianças.

Ao associar o sexo com a classificação motora, evidenciou-se que os meninos relataram uma tendência a ter dificuldade motora. Dados da Organização Mundial de Saúde (ORGANIZAÇÃO..., 1993) indicam que este transtorno ocorre com maior frequência em meninos, numa proporçáo de quatro ou cinco meninos para cada menina. Miranda, Beltrame e Cardoso (2011) avaliaram 380 escolares em Florianópolis-SC e o sexo masculino apresentou maior número de casos de TDC do que o sexo feminino, mostrando uma relação de quase 4:1. Green et al. (2011), investigando 4.331 crianças inglesas, identificaram 193 delas com provável TDC, sendo $60,6 \%$ meninos e $40,4 \%$ meninas. Ellinoudiset, Kiparisis e Kourtesiset (2009) encontraram 59 crianças gregas com TDC, das 330 avaliadas, sendo 52,0\% meninos. Teixeira et al. (2010) obtiveram prevalência significativa de meninos com TDC em escolas públicas, sendo que, nas escolas privadas, as meninas apresentaram maior prevalência; porém, esta diferença não foi significativa. De acordo com Toniolo e Capellini (2010), na faixa etária entre 6 e 13 anos, há uma maior proporção de meninos identificados com TDC, o que vem a ser confirmado por Gibbs, Appleton e Appleton (2007), os quais afirmam que os meninos demonstram quatro vezes mais chance de apresentar o transtorno em relação às meninas. Alguns fatores que podem implicar nas frequências distintas entre meninos e meninas com TDC são as dificuldades apresentadas por eles nas atividades que exigem coordenação motora fina (tarefas de destreza manual para os meninos e habilidades com bola para as meninas), que poderiam estar interferindo no resultado total das avaliações motoras e reduzindo os escores totais do teste. 
Tal fato pode ser comprovado ao se verificarem os escores superiores para as meninas nos domínios de destreza manual e equilíbrio, e para os meninos, no lançar e receber, verificados no presente estudo. Ainda, o contexto cultural no qual a criança está inserida influencia seu desempenho, bem como as oportunidades de experiências motoras, já que os meninos, culturalmente, são incentivados a atividades mais vigorosas e que exijam coordenaçáo ampla, não sendo tão desenvolvidas as habilidades finas, justificando assim a menor pontuação no domínio destreza manual, enquanto que as meninas optam por atividades mais calmas, como brincar com bonecas, desenhar, entre outras (PELLEGRINI et al., 2008; COUTINHO; SPESSATO; VALENTINI, 2011).

Em relação ao IMC, as crianças com risco e dificuldade motora apresentaram médias superiores para o IMC quando comparadas às crianças sem dificuldade. Esses resultados vão ao encontro de estudos internacionais que apontam índices elevados de sobrepeso para crianças com TDC. Em Taiwan, 2.057 crianças de 9 a 12 anos de idade foram avaliadas com o MABC-2: os participantes do estudo com TDC foram significativamente mais propensos a serem obesos quando comparados com crianças sem TDC (ZHU et al., 2014). No Canadá, um estudo longitudinal acompanhou, por dois anos, 2.278 crianças, sendo que 111 tinham o TDC; essas crianças apresentaram maior média de IMC e circunferência da cintura no início do estudo do que aquelas sem dificuldade motora. Essas diferenças persistiram ou aumentaram ligeiramente ao longo do tempo. De forma geral, os autores encontraram que crianças com TDC apresentaram três vezes mais chance de ter sobrepeso (OR: 3,44, IC 95\%: 2,34-5,07) e quatro vezes mais chance de apresentar obesidade (OR: 4,00, IC 95\%: 2,57-6,21) em relação àquelas sem o transtorno (CAIRNEY et al., 2010). Na Holanda, um estudo de revisão sistemática, realizado em seis bases de dados, concluiu que as crianças com TDC apresentaram maiores escores para o IMC, a circunferência da cintura e a porcentagem de gordura corporal em comparação com os escolares sem o transtorno (HENDRIX; PRINS; DEKKERS, 2014). No Canadá, outro estudo de revisão sistemática encontrou 18 manuscritos que avaliaram a composição corporal e verificaram os efeitos da mesma na proficiência motora de crianças, e 13 destes evidenciaram que a coordenação motora pobre leva a um aumento de IMC, peso corporal e percentual de gordura relativa (RIVILLIS et al., 2011). Em relação aos estudos nacionais, em Florianópolis-SC, foram avaliados 380 escolares de 7 a 10 anos, e náo houve associação entre o estado nutricional e o transtorno (MIRANDA; BELTRAME; CARDOSO, 2011). Em Farroupilha-RS, 287 crianças de 5 a 10 anos foram avaliadas, sendo que as crianças obesas e com desnutrição apresentaram índices maiores de desenvolvimento motor pobre (MARRAMARCO et al., 2012). As diferenças encontradas pelos estudos podem ser explicadas pelos distintos procedimentos metodológicos utilizados em cada investigação.

Quanto aos aspectos que contribuem para a associação encontrada entre o IMC e a classificação motora no presente estudo, Rivillis et al. (2011) pontuam a reduzida participação de crianças com TDC em atividades físicas livres ou planejadas, e a baixa percepção de competência e autoestima, que acentua a hipoatividade e leva à diminuição da energia consumida e ao aumento da gordura corporal. Os autores também observaram evidências de que, mesmo que a relação entre os baixos níveis de atividade física e o aumento da massa corporal também ocorra em crianças com desenvolvimento típico, essa relação é bem mais acentuada em crianças com déficits na coordenação motora. Diante dessas informações, salienta-se a necessidade de maior atenção ao dia a dia das crianças com TDC, incentivando a prática de atividade física de maneira prazerosa, a fim de que este estilo de vida ativo seja implantado no cotidiano dessas crianças.

Em relação à resistência cardiorrespiratória, as crianças com risco e dificuldade motora apresentaram menor resistência do que as crianças sem dificuldade motora. Note-se que crianças com resistência cardiorrespiratória menor que $654 \mathrm{~m}$ estão mais propensas para desenvolverem dificuldade motora. Resultados semelhantes foram encontrados na África do Sul, em que, das 140 crianças avaliadas, 70 apresentaram TDC, demonstrando uma capacidade aeróbica menor do que as sem TDC (FERGUSON et al., 2014). Pesquisadores da Holanda avaliaram 76 crianças com idades entre 7 e 12 anos, sendo 38 participantes com TDC; os resultados apontaram que a capacidade aeróbica foi significativamente menor para as crianças com dificuldade motora e os autores enfatizam que atividades físicas adequadas devem ser incentivadas em crianças com dificuldade motora, a fim de reverter este quadro (OUDENAMPSEN et al., 2013). Van-Der et al. (2012) investigaram 76 crianças holandesas, sendo 38 destas com TDC. Foram encontrados valores significativamente menores de aptidão cardiorrespiratória máxima em 6,7\% das crianças que apresentaram o transtorno. Já no estudo de Haga (2008), ao avaliar a aptidão física 
relacionada à saúde em crianças de 9 e 10 anos, com e sem dificuldades motoras, foram verificadas diferenças significativas entre os grupos para todas as nove tarefas do Physical Best, com pior performance sendo apresentada pelo grupo com dificuldades motoras.

Conforme alguns autores, o fraco desempenho das crianças com TDC pode ocorrer devido à restrita participação em atividades físicas, que é acentuada pela coordenação motora pobre (CAIRNEY et al., 2005, 2010; TSIOTRA et al., 2006; HAGA, 2008; WU et al., 2010). Muitas investigaçôes têm averiguado que crianças com TDC se engajam menos em atividades vigorosas, como correr e saltar, e ainda têm menor participação em atividades organizadas, como jogos com regras, o que contribui para os baixos escores nas avaliaçóes de aptidão física, resultando em maiores riscos para o desenvolvimento de sobrepeso/obesidade (TSIOTRA et al., 2006; HAGA, 2008; CAIRNEY et al., 2010; WU et al., 2010; ZHU; WU; CAIRNEY, 2011). As poucas vivências motoras e a falta de envolvimento com atividades físicas atuam como uma barreira ao desenvolvimento das aptidóes físicas necessárias para a realização de atividades vigorosas na vida diária e também no lazer e nos esportes; dentre estas aptidóes, encontram-se as condiçóes de força, flexibilidade, resistência cardiorrespiratória (HAGA, 2008).

\section{Conclusão}

De acordo com os resultados do presente estudo, pode-se afirmar que os meninos apresentaram maiores prevalências de risco/dificuldade motora em relação às meninas. Os escolares com TDC demonstraram IMC superior e resistência cardiorrespiratória inferior, quando comparados aos seus pares sem o transtorno. Além disso, verificou-se que as crianças com dificuldades motoras estáo propensas a apresentar uma baixa resistência cardiorrespiratória, inferior ao ponto de corte de $654 \mathrm{~m}$.

Destacamos a necessidade de atenção ao grupo de alunos classificados com risco ou dificuldade significativa de movimento, pois esses resultados podem orientar pais, professores e outros profissionais da saúde sobre a importância da elaboração e da aplicação de estratégias para compensar as dificuldades motoras, bem como oferecer oportunidades para potencializar e estimular as capacidades motoras, além da conscientização sobre a atividade física na vida das crianças.

\section{Referências}

AMERICAN PSYCHIATRIC ASSOCIATION - APA. Manual Diagnóstico e Estatístico de Transtornos Mentais DSM-V. Porto Alegre: Artmed, 2014.

ASSOCIAÇÃO BRASILEIRA DE EMPRESAS DE PESQUISA - ABEP. Critério de classificação econômica Brasil. São Paulo: ABEP, 2012. Disponível em: <http:// www.abep.org/criterio-brasil>. Acesso em: 20 out. 2013.

ALVAREZ, B. R.; PAVAN, A. L. Alturas e comprimentos. In: PETROSKI, E. L. Antropometria: técnicas e padronizaçóes. Porto Alegre: Pallotti, 1999. p. 29-51.

BARROS, M. V. G. et al. Validity of physical activity and food consumption questionnaire for children aged seven to ten years old. Revista Brasileira de Saúde Materno Infantil, Recife, v. 7, n. 4, p. 437-448, 2007. http:// dx.doi.org/10.1590/S1519-38292007000400011.

BENEDETTI, T. R. B.; PINHO, R. A.; RAMOS, V. M. Dobras cutâneas. In. PETROSKI, E. L. Antropometria: técnicas e padronizações. Porto Alegre: Pallotti, 1999. p. 53-67.

CAIRNEY, J. et al. Developmental coordination disorder, self-efficacy toward physical activity, and play: does gender matter? Adapted Physical Activity Quarterly, Champaign, v. 22, n. 1, p. 67-82, 2005. Disponível em: $<$ http://epe.sagepub.com/content/13/1/81.refs $>$. Acesso em: 5 mar. 2013.

CAIRNEY, J. et al. Developmental coordination disorder, sex, and activity deficit over time: a longitudinal analysis of participation trajectories in children with and without coordination difficulties. Developmental Medicine and Child Neurology, Newcastle, v. 52, n. 3, p. e67-e72, 2010. http://dx.doi.org/10.1111/j.14698749.2009.03520.x. PMid:20015253.

CAIRNEY, J. et al. Developmental Coordination Disorder, gender, and body weight: examining the impact of participation in active play. Research in Developmental Disabilities, London, v. 33, n. 5, p. 1566-1573, 2012. http://dx.doi.org/10.1016/j.ridd.2012.02.026. PMid:22522216.

COUTINHO, M. T. C.; SPESSATO, B. C.; VALENTINI, N. C. Transtorno do desenvolvimento da coordenaçăo: prevalência e dificuldades motoras de escolares da cidade de Porto Alegre. In: CONGRESSO BRASILEIRO DE CIÊNCIAS DO ESPORTE, 17., 2011, Porto Alegre. Anais... Porto Alegre: CBCE, 2011. p. 1-9. Disponível em: <http://congressos.cbce.org.br/index.php/ conbrace2011/2011/paper/view/3292>. Acesso em: 10 nov. 2013.

ELLINOUDIS, T.; KIPARISIS, M.; KOURTESISET, T. H. Identification of children aged 7-12 with developmental coordination disorder by physical education teachers using the test "Movement Assessment Battery for Children". Hellenic Journal of Physical Education e Sport Science, Greece, v. 29, n. 3, p. 288-306, 2009. Dispo- 
nível em: <http://www.hellenicjsport.com/en_mission. html>. Acesso em: 5 mar. 2013.

FERGUSON, G. D. et al. Physical fitness in children with Developmental Coordination Disorder: measurement matters. Research in Developmental Disabilities, Oxford, v. 35, n. 5, p. 1087-1097, 2014. http://dx.doi. org/10.1016/j.ridd.2014.01.031. PMid:24582141.

FRANÇA, C. Desordem coordenativa desenvolvimental em crianças de 7 e 8 anos de idade. 2008. 95 f. Dissertação (Mestrado em Ciências do Movimento Humano) - Universidade do Estado de Santa Catarina, Florianópolis, 2009.

GAYA, A. C. A. Projeto Esporte Brasil-PROESP BRASIL: Manual de aplicaçáo de medidas e testes, normas e critérios de avaliação. Porto Alegre: UFRGS, 2009.

GIBBS, J.; APPLETON, J.; APPLETON, R. Dyspraxia or developmental coordination disorder? Unravelling the enigma. Archives of Disease in Childhood, London, v. 92, n. 6, p. 534-539, 2007. http://dx.doi.org/10.1136/ adc. 2005.088054 .

GREEN, D. et al. The risk of reduced physical activity in children with probable Developmental Coordination Disorder: a prospective longitudinal study. Research Developmental Disabilities, Oxford, v. 32, n. 4, p. 1332-1342, 2011. http://dx.doi.org/10.1016/j. ridd.2011.01.040. PMID: 21334850.

HAGA, M. Physical fitness in children with movement difficulties. Physiotherapy, Oxford, v. 94, n. 3, p. 253-259, 2008. http://dx.doi.org/10.1016/j.physio.2007.04.011.

HANDS, B.; LARKIN, D. Physical fitness differences in children with and without "motor learning difficulties". European Journal of Special Needs Education, London, v. 21, n. 4, p. 447-456, 2006. http://dx.doi. org/10.1080/08856250600956410.

HENDRIX, C. G.; PRINS, M. R.; DEKKERS, H. Developmental coordination disorder and overweight and obesity in children: a systematic review. Obesity Reviews, Malden, v. 15, n. 5, p. 408-423, 2014. http://dx.doi. org/10.1111/obr.12137. PMid:24387283.

MARRAMARCO, C. A. et al. Crianças desnutridas pregressas, com sobrepeso e obesas apresentam desempenho motor pobre. Revista de Educação Física/UEM, Maringá, v. 23, n. 2, p. 175-182, 2012.

MARTINS, M. O.; LOPES, M. A. Perímetros. In: PETROSKI, E.L. Antropometria: técnicas e padronizaçóes. Porto Alegre: Pallotti, 2003. p. 59-71.

MIRANDA, T. B.; BELTRAME, T. S.; CARDOSO, F. L. Desempenho motor e estado nutricionsl de escolares com e sem transtorno do desenvolvimento da coordenação. Revista Brasileira de Cineantropometria e Desempenho Humano, Florianópolis, v. 13, n. 1, p. 59-66, 2011.

ORGANIZAÇÃO MUNDIAL DE SAÚDE - OMS. Classificação dos transtornos mentais e de comportamento da CID-10: descriçôes clínicas e diretrizes diagnósticas. Porto Alegre: Artes Médicas, 1993.

OUDENAMPSEN, C. et al. Relationship between participation in leisure time physical activities and aerobic fitness in children with DCD. Pediatric Physical Therapy, Philadelphia, v. 25, n. 4, p. 422-429, 2013. http://dx.doi.org/10.1097/PEP.0b013e3182a6b6ea. PMid:24076629.

PAYNE, V. G.; ISAACS, L. D. Desenvolvimento motor humano: uma abordagem vitalícia. Rio de Janeiro: Guanabara Koogan, 2007.

PELLEGRINI, A. M. et al. Dificuldades motoras em crianças de 9-10 anos de idade: seriam os meninos mais descoordenados? In: PINHO, S. Z.; SAGLIETTI, J. R. C. Núcleos de ensino da UNESP. São Paulo: Cultura Acadêmica, 2008. p. 77-88. Disponível em: <http:// www. unesp.br/prograd/PDFNE2006/artigos/.../dificuldades. pdf>. Acesso em: 20 mar. 2013.

RIVILLIS, I. et al. Physical activity and fitness in children with developmental coordination disorder: systematic review. Research in Developmental Disabilities, New York, v. 32, n. 3, p. 894-910, 2011. http://dx.doi. org/10.1016/j.ridd.2011.01.017. PMID: 21310588.

SILVA, J.; BELTRAME, S. T. Indicativo de transtorno do desenvolvimento da coordenaçáo de escolares com idade entre 7 e 10 anos. Revista Brasileira de Ciências do Esporte, Florianópolis, v. 35, n. 1, p. 3-14, 2013. http:// dx.doi.org/10.1590/S0101-32892013000100002.

SLAUGHTER, M. H. et al. Skinfold equations for estimation of body fatness in children and youth. $\mathrm{Hu}$ man Biology, Malden, v. 60, n. 5, p. 709-723, 1988. PMid:3224965.

TEIXEIRA, R. et al. Dificuldades motoras na infância: prevalência e relaçóes com as condiçóes sociais e econômicas. Science in Health, São Paulo, v. 1, n. 1, p. 25-34, 2010.

TONIOLO, C. S.; CAPELLINI, S. A. Transtorno do desenvolvimento da coordenação: revisão de literatura sobre instrumentos de avaliação. Revista Psicopedagogia, Sáo Paulo, v. 27, n. 82, p. 109-116, 2010.

TSIOTRA, G. D. et al. A comparison of developmental coordination disorder prevalence rates in Canadian and Greek children. Journal of Adolescent Health, San Francisco, v. 39, n. 1, p. 125-127, 2006. Disponível em: <http://www.jahonline.org/article/S1054-139X\%2805\%2900374-5/abstract>. Acesso em: 18 jul. 2013.

VAN-DER, H. F. D. et al. Health-related physical fitness in Dutch children with developmental coordination disorder. Journal of Developmental \& Behavioral Pediatrics, Philadelphia, v. 33, n. 8, p. 649-655, 2012. http://dx.doi.org/10.1097/DBP.0b013e3182653c50. PMid:23027139.

ZHU, Y.; WU, S. K.; CAIRNEY, J. Obesity and motor coordination ability in Taiwanese children with 
and without developmental coordination disorder. Research in Developmental Disabilities, New York, v. 32, n. 2, p. 801-807, 2011. http://dx.doi.org/10.1016/j. ridd.2010.10.020. PMID: 21109392.

ZHU, Y. C. et al. High risk for obesity in children with a subtype of developmental coordination disorder. Research in Developmental Disabilities, Oxford, v. 35, n. 7, p. 1727-1733, 2014. http://dx.doi.org/10.1016/j. ridd.2014.02.020. PMid:24685095.
WANG, Y.; MONTEIRO, C.; POPKIN, B. M. Trends of obesity and underweight in older children and adolescents in the United States, Brazil, China and Russia. The American Journal of Clinical Nutrition, Bethesda, v. 75, n. 6, p. 971-977, 2002. PMid:12036801.

WU, S. K. et al. Cardiopulmonary fitness and endurance in children with developmental coordination disorder. Research Developmental Disabilities, New York, v. 31, n. 2, p. 345-349, 2010. http://dx.doi.org/10.1016/j. ridd.2009.09.018. PMID: 19913384.

\section{Contribuição dos Autores}

Renata Capistrano realizou coleta, organização de fontes, análise e redação do texto. Juliano Maestri Alexandre, organização de fontes, análise e redação do texto. Thais Silva Beltrame, análise e revisão do texto. Todos os autores aprovaram a versão final do texto.

\section{Fonte de Financiamento}

Coordenação de Aperfeiçoamento de Pessoal de Nível Superior (CAPES). 